\title{
ETUDES SUR LES PARASITES DE L'HUITRE COMESTIBLE DU JAPON OSTREA GIGAS THUNBERG
}

\author{
Par TSUNENOBU FUJITA
}

\author{
Traduction accompagnée de notes, de diagnoses \\ ET D'UNE BIBLIOGRAPHIE \\ Par Robert-Ph. Dollfus
}

Deux espèces de trématodes parasites ont été trouvées dans l'huître commune du Japon : Ostrea gigas Thunberg. L'une est parasite à la surface externe du manteau, les individus étant généralement rassemblés par groupes; elle était déjà connue des ostréiculteurs. L'autre, découverte en 1904 par l'auteur, est parasite dans la glande génitale ; les individus s'y trouvent séparément, au sein des tissus (1).

\section{A. Forme parasite à la surface du manteau.}

Metacercaria : Gymnophalloides tokiensis, n. g. (2), n. sp. (fig. 1-3)

Le corps est linguiforme, très légèrement en pointe à son extrémité postérieure. Il mesure $0 \mathrm{~mm}$., 36 de long sur $0 \mathrm{~mm}$., 23 de large en son milieu. Le tégument est épais d'environ $0 \mathrm{~mm}$., 004 et sa surface est ornée de rangées de nombreuses petites ponctuations en quinconces. Sur des coupes on voit qu'il est pourvu de nombreuses fines striations, en plus des petites élévations que forment les ponctuations (3). Les deux ventouses sont asymétriques et situées assez loin l'une de l'autre. La ventouse orale, subterminale, est plus grande, oblongue, allongée transversalement, elle mesure $0 \mathrm{~mm}$., 13

(1) M. T. Fujita nous a aimablement communiqué des préparations de ces deux parasites en nous priant de les déterminer génériquement; nous avons reconnu que ces deux parasites, appartenant à des genres différents, étaient des larves au stade metacercaria. - R.-Ph. D.

(2) Nous proposons provisoirement le genre nouveau Gymnophalloides, pour des motifs que nous exposerons plus loin. - R.-Ph. D.

(3) Les ponctuations en relief sont infiniment plus serrées et nombreuses que ne permet de le supposer la figure 1, demi schématique. - R.-Ph. D.

Annales de Parasitologie, T. III, No 1 . - Janvier 1925, p. 37-59. 
sur $0 \mathrm{~mm} ., 09$ (1). La ventouse ventrale est petite, presque sphérique, mesurant environ $0 \mathrm{~mm}$., 05 sur $0 \mathrm{~mm}$., 06 , elle est située à la limite des deux derniers tiers de la longueur totale du corps. La paroi de ces ventouses est généralement épaisse de $0 \mathrm{~mm}$., 015 et limitée intérieurement par une mince cuticule. Sur des coupes, elle apparaît complètement lisse, ce qui contraste avec la paroi du corps immédiatement voisine.

La bouche s'ouvre dans le fond de la ventouse orale, qui est profonde d'environ $0 \mathrm{~mm}$., 01 . Le pharynx, qui lui fait immédiatement suite, est relativement petit, sphérique, long de $0 \mathrm{~mm}$., 02 ; il est suivi d'un œsophage ayant environ la même longueur, mais plus étroit. Les branches de l'intestin forment deux sacs renflés, plus ou moins divergents, dans la région moyenne centrale du corps. Leur extrémité postérieure dépasse généralement le niveau de la moitié de la longueur du corps. Ces sacs intestinaux mesurent $0 \mathrm{~mm}$., 08 sur $0 \mathrm{~mm}$., 04. Leur paroi est formée de très grandes cellules, hautes de $0 \mathrm{~mm}$., 004, généralement pourvues d'une proéminence en dedans et souvent vacuolaires antérieurement. La vessie est en forme de $\mathrm{V}$ avec des parois à contours non rectilignes, irréguliers.

Antérieurement, ses branches atteignent la ventouse orale, au niveau de laquelle elles se dilatent quelque peu latéralement, puis, devenant beaucoup plus étroites, elles prennent une direction postérieure et, se rapprochant de la face dorsale, longent latéralement chaque côté du corps. Dans cette partie de leur parcours, les branches excrétrices émettent irrégulièrement dans toutes les directions de nombreux rameaux. La paroi de la vessie est très mince et pourvue çà et là de noyaux. Dans les rameaux, on observe distinctement des grains d'excrétion.

Il existe une paire de taches oculaires sphériques, au niveau de lovaire, du côté ventral (2).

L'appareil génital est presque entièrement confiné dans le tiers postérieur du corps et apparaît comme encore immature, ce qui rend difficile à discerner la véritable nature des parties qui le composent. Le pore génital est bien apparent, il a la forme d'une large fente transversale, longue de $0 \mathrm{~mm}$., 02 env.; il occupe, au milieu de la face ventrale, un niveau correspondant presque exactement à la limite postérieure des cæca intestinaux. En coupe, il se pré-

(1) Cette ventouse est très puissante, quand on enlève la métacercaire du manteau elle retient dans sa cavité des faisceaux de cellules épithéliales arrachées, ainsi que nous l'avons reconnu sur des préparations montrant le parasite in situ - R.-Ph. D.

(2) Remarquons que la position ventrale decette paire de taches oculaires est une exception chez les Digenea, où elles sont, en général, en connectiondirecte avec les troncs nerveux dorsaux (postérieurs ou antérieurs). Nous n'avons pu retrouver ces organes sur les préparations colorées que nous avons examinées. - R.-Ph. D. 


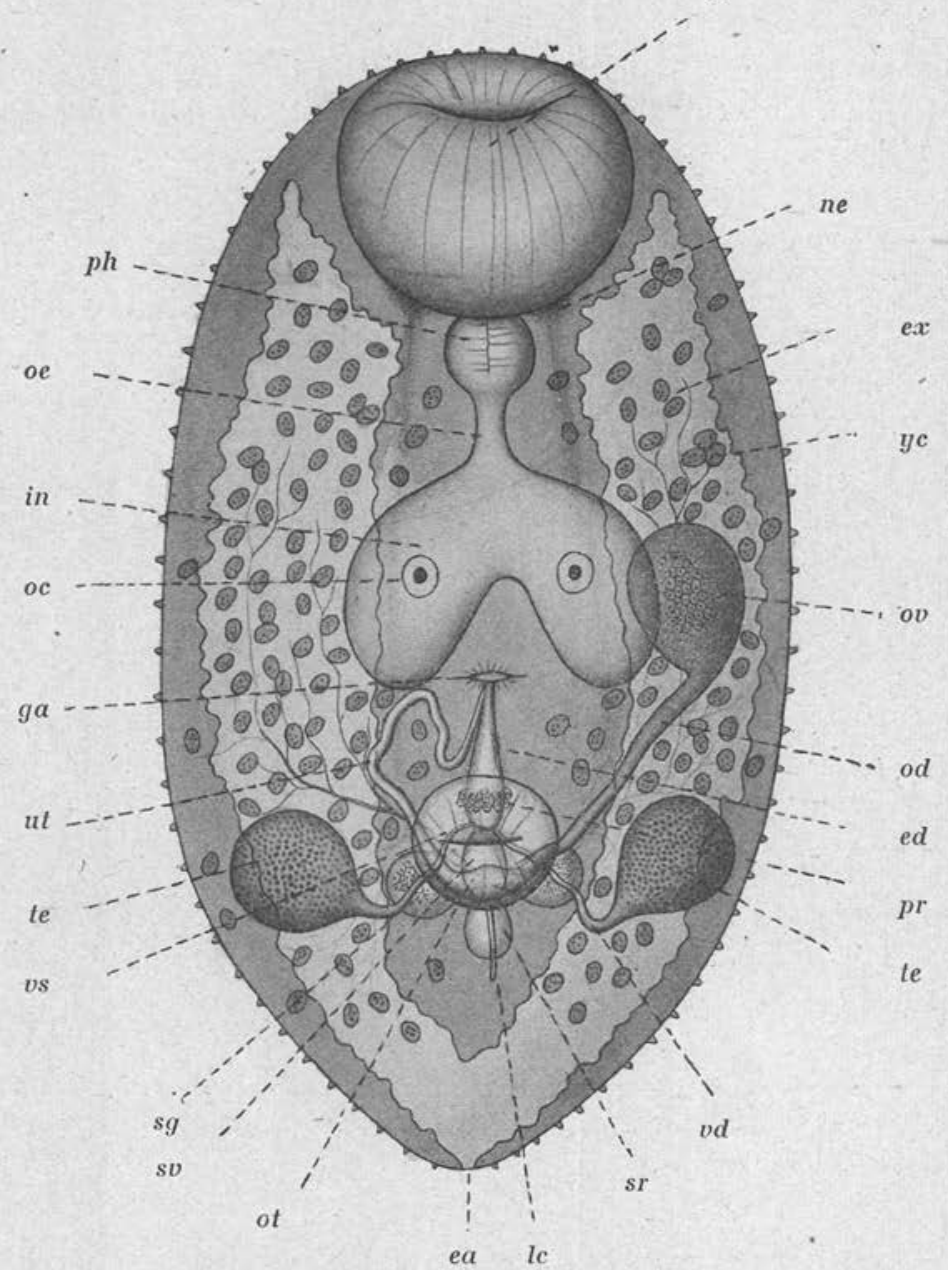

FIG. 1. - Metacercaria: Gymnophalloides tokiensis n. g., n. sp. individu in toto (demi-diagrammatique), $\times$ env. 360 (1).

$c a$, canal excréteur (?) $e d$, canal éjaculateur.

ga, orifice génital.

ne, système nerveux central.

$\propto$, œsophage.

$o v$, ovaire.

$p g$, glande prostatique.

$s m$, sphincter.

te, testicule.

$v d$, canal déférent. $c b$, poche du cirrhe.

$e x$, vessie.

$i n$, branche intestinale.

$o c$, tache oculaire.

$o s$, ventouse orale.

pe, pénis.

$p r$, prostate.

$s v$, vésicule séminale.

$u t$, utérus.

$y c$, cellule vitellogène. $e a$, orifice de la vessie.

$f c$, crête fibreuse.

$l c$, canal de Laurer.

od, oviducte.

ot, ootype.

ph, pharynx.

$s g$, glande de Mehlis.

$s r$, receptaculum seminis. $v s$, ventouse ventrale. $y d$, vitelloducte.

(1) Nous ne sommes pas toujours d'accord avec Fujita pour l'interprétation des organes, ainsi qu'il a été dit précédemment; pour nous, par exemple, les organes marqués $s g$ sur les figures $1,2,3$, ne représentent pas des glandes de Mehlis mais bien des vitellogènes; les cellules marquées yc sur les figures $1,2,4$, ne représentent pas des cellules vitellogènes, mais des glandes unicellulaires du parenchyme, etc... - R.-Ph. D. 
sente sous la forme d'un entonnoir dont la large ouverture correspond à la face ventrale (figure $2 g a$ ), il est intérieurement garni d'une épaisse cuticule fortement plissée. Le pourtour de son bord antéro-externe est pourvu d'un anneau musculaire proéminent (sphincter).

Il y a deux testicules, presque symétriques, chacun d'un côté de la ventouse ventrale; ils sont bien plus postérieurs que l'ovaire et

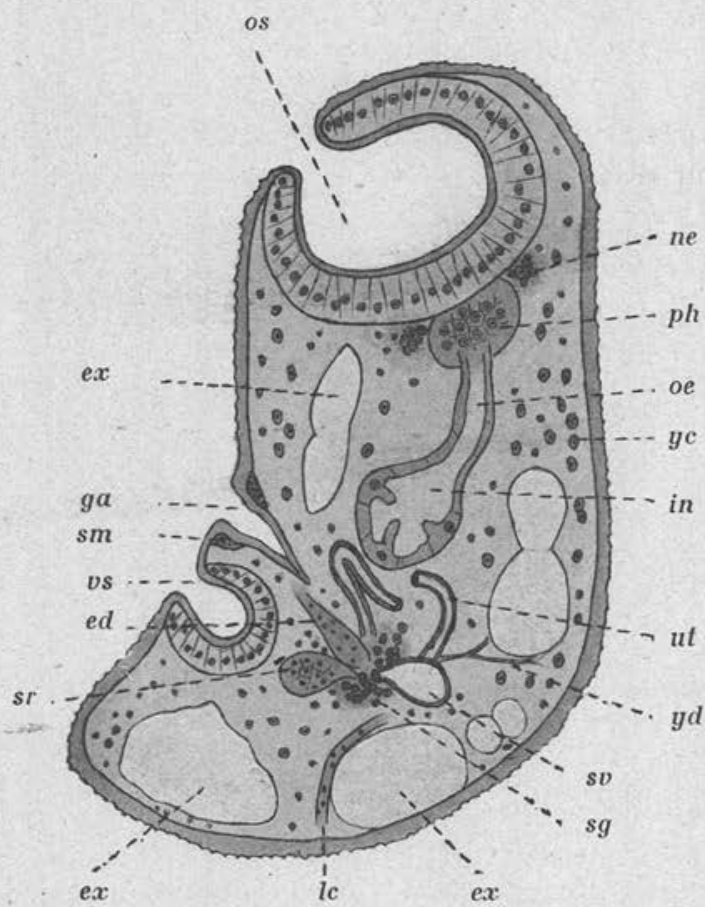

Fig. 2. - Metacercaria : Gymnophalloides tokiensis. Coupe longitudinale, $\times 220$ (mêmes lettres que fig. 1).

en même temps plus rapprochés de la face ventrale. Ils sont également de forme ovale et mesurent $0 \mathrm{~mm}$., 025 dans leur plus grande longueur. De leur extrémité postéro-dorsale part chaque vas deferens, tubes très fins à paroi renfermant de nombreux noyaux. Près de leur origine, ils se dirigent de dehors en dedans, en se rapprochant de la face dorsale, vers la ligne médiane du corps ; puis, changeant de direction, montrent un trajet ascendant vers la face ventrale, jusque du côté dorsal de la ventouse ventrale, où ils se réunissent et s'ouvrent dans la vésicule séminale en sa partie postéro-dorsale. 
La vésicule séminale est un sac ovale à paroi mince, nuclée, qui ressemble beaucoup, tant par sa forme que par sa taille, au receptaculum seminis. On voit, sur les coupes, que ces deux organes sont situés au même niveau, mais dans des régions différentes du corps : la vésicule appartient à la région dorsale, le réceptacle à la région ventrale. Il n'y a pas de poche du cirre. Cependant, juste en avant de la vésicule séminale, il existe, en position médio-sagittale, un sac claviforme, dilaté dans sa partie proximale, et dirigé, obliquement et en avant, de la région dorsale à la région ventrale du corps. Il mesure environ $0 \mathrm{~mm}$., 05 de long sur $0 \mathrm{~mm}$., 005 dans sa plus grande largeur. Sa paroi est très mince et contient des noyaux bien distincts. Autour de la partie dilatée de ce sac sont groupées de nom-

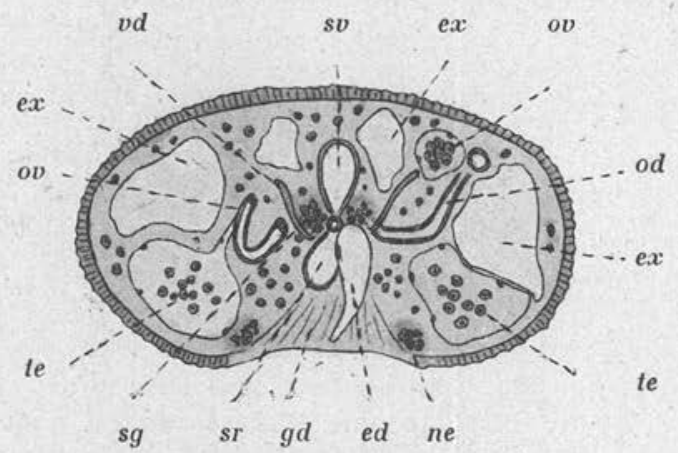

Fig. 3. - Metacercaria : Gymnophalloides tokiensis. Section transversale montrant une partie de l'appareil génital, $\times 390$ (mêmes lettres que fig. 1).

breuses cellules prostatiques, montrant que ce sac représente la " pars prostatica ". La portion antérieure de ce sac est plus ou moins prolongée par un tube mince dont la paroi devient plus épaisse que celle du sac et qui, par son extrémité antérieure, aboutit au pore génital ; il correspond au canal éjaculateur. L'ovaire est situé presque entièrement du côté dorsal gauche de la partie moyenne du cæcum intestinal gauche et à son niveau, il est distant de $0 \mathrm{~mm}$., 18 de l'extrémité antérieure du corps. Il a à peu près la même forme et les mêmes dimensions que les testicules ; sa paroi est semblablement très mince. L'oviducte part de sa partie postéroexterne et descend par un trajet postéro-dorsal vers la ventouse ventrale. Il est relativement court et plutôt large, mesurant approximativement $0 \mathrm{~mm}$., 05 de long avec un diamètre de $0 \mathrm{~mm}$., 008 . Il s'élargit dans la région médiane du corps, son diamètre atteignant $0 \mathrm{~mm}$., 012, formant un ootype sensiblement sphérique. Du côté ventral de l'ootype part l'utérus, il est un peu plus long ( $0 \mathrm{~mm}$., 08 ) 
que l'oviducte, mais plus étroit (sa lumière mesurant environ $0 \mathrm{~mm} ., 005$ ). Tout d'abord l'utérus se dirige ventralement en avant, puis, près de l'extrémité postérieure du cæcum intestinal droit, il se détourne brusquement vers l'arrière le long de la face dorsale du corps, sur une longueur égale à la moitié de son trajet, pour finalement aboutir au pore génital à gauche de l'organe éjaculateur. Le "receptaculum seminis " est un sac ovale de $0 \mathrm{~mm}$., 002, qui s'ouvre dans l'ootype, du côté ventral de l'ootype (1). De l'extrémité dorsale de ce sac, part le canal de Laurer qui est très nettement. visible et relativement court. Il a un trajet postéro-dorsal et s'ouvre à la face dorsale du corps à une distance d'environ $0 \mathrm{~mm}$., 006 de l'extrémité postérieure. Les glandes de Mehlis forment, de chaque côté de l'ootype, une masse compacte, pyramidale, de $0 \mathrm{~mm}$., 15 sur $0 \mathrm{~mm}$., 05 ; par leur étroit apex, elles se réunissent et s'ouvrent dans l'ootype (2).

Les vitellogènes sont représentés par des cellules petites et peu apparentes, distribuées le long des côtés du corps, en avant du niveau de la ventouse orale et en arrière de l'extrémité antérieure de l'organe excréteur. Les vitelloductes ont une direction presque horizontale et s'ouvrent dans l'ootype.

Remarque. - Ce distome est plus généralement trouvé dans les huîtres des fonds vaseux, des eaux à salinité peu élevée telles que celles de la baie de Tokyo et de la baie d'Isé. Quelques années auparavant, j’ai récolté de nombreuses huîtres de différents districts, du nord d'Hokkaido et du sud de Kiushu, connus pour produire de bonnes huitres marchandes. Après une minutieuse recherche chez ces huîtres, j'ai été convaincu que les exemplaires des deux baies ci-dessus mentiónnées montraient une contamination notable par ce parasite, alors que les autres exemplaires,

(1) Doit-on interpréter réellement ce sac comme un \& receptaculum seminis » ou peut-il être interprété différemment si l'on suppose qu'il n'y a pas, comme chez les Gymnophallus, de " receptaculum seminis »? Nous restons très hésitants à ce sujet car ni sur des spécimens in toto, ni sur des coupes nous n'avons pu nous en rendre compte. Nous nous étions même à un moment demandé si la vésicule séminale n'était pas, comme chez Gymnophallus, divisée en deux parties par une constriction; s'il en était ainsi, le a receptaculum seminis ) de Fujita serait en réalité la partie la plus ventrale de la vésicule séminale. Nous regrettons que nos matériaux ne se soient pas prêtés à une étude précise de cette partie de l'appareil génital. - R.-Ph. D.

(2) Les deux amas glandulaires que Fujita considère comme glandes de Mehlis sont certainement, selon nous, les vitellogènes, et ils occupent la même situation chez les Gymnophallus. Les grandes cellules glandulaires dispersées dans le parenchyme de presque tout le corps, que Fujita décrit comme étant des follicules vitellogènes, ne nous paraissent pas pouvoirêtre considérées comme tels. De semblables éléments existent dans le parenchyme de métacercaires de Gymnophallus; par exemple chez Metacercaria Gymnophallus glandosa M. V. Lebour (1908, p. 4-5, 20, pl. I, fig. 1 ; 1912 , p. 428, 454, pl. XXV, fig. 10), des Paludestrina stagnalis Baster du Northumberland; toutefois, chez cette espèce, ils sont cantonnés dans la région antérieure du corps. Nous avons personnellement observé des cellules glandulaires du même type dans le parenchyme de la métacercaire parasite des Donax vittatus Da Costa, de Boulogne-sur-Mer, que nous avons cru, en 1912, pouvoir rapporter à Metacercaria Gymnophallus strigata M. V. Lebour. - R.-Ph. D. 
de tous les autres districts, en étaient indemnes. Ce fait est clairement illustré par le tableau suivant:

TABleaU I. - Nombre de distomes trouvés dans les huîtres de différents districts

\begin{tabular}{|c|c|c|c|c|c|c|}
\hline \multirow{3}{*}{ Districts } & \multicolumn{2}{|c|}{$\begin{array}{c}\text { NoMbRe } \\
\text { D'HUITRES }\end{array}$} & \multirow{2}{*}{\multicolumn{2}{|c|}{\begin{tabular}{|c|} 
NOMBRE \\
D'HUITRES REN- \\
FERANANT.DES \\
PARASITES SUR \\
LE MANTEAU \\
DU CÔTÉ
\end{tabular}}} & \multirow{2}{*}{\multicolumn{2}{|c|}{$\begin{array}{l}\text { NOMBRE MOYEN } \\
\text { DE DISTOMES } \\
\text { DANS UNE HUITRE } \\
\text { CÔTÉ }\end{array}$}} \\
\hline & \multirow[t]{2}{*}{ examinees } & \multirow[t]{2}{*}{ parasitées } & & & & \\
\hline & & & droit & gauche & droit & gauche \\
\hline Akkeshi (Hokkaido). & 20 & 0 & 0 & 0 & 0 & 0 \\
\hline Saruma (Hokkaido)............ & 20 & 0 & 0 & 0 & 0 & 0 \\
\hline Matsushima (Matsushima Bay).. & 30 & 0 & 0 & 0 & 0 & 0 \\
\hline Matsukawa (Matsukawa Bay)... & 30 & 0 & 0 & 0 & 0 & 0 \\
\hline Futtsu (Tokyo Bay)............. & 20 & 18 & 4 & 18 & 15 & 30 \\
\hline Kisarazu (Tokyo Bay) .......... & 20 & 18 & 6 & 18 & 35 & 51 \\
\hline Narawa (Tokyo Bay)........... & 20 & 20 & 0 & 20 & 0 & 65 \\
\hline Yawata (Tokyo Bay)........... & 20 & 20 & 20 & 20 & 53 & 107 \\
\hline Shinagawa (Tokyo Bay)........ & 20 & 220 & 20 & 20 & 250 & 724 \\
\hline Omori (Tokyo Bay)............ & 20 & 20 & 20 & 20 & 218 & 645 \\
\hline Daishigawara (Tokyo Bay) ..... & 20 & 19 & 3 & 19 & 2 & 50 \\
\hline Kanagawa (Tokyo Bay)......... & 20 & 14 & 2 & 14 & 3 & 78 \\
\hline Yokohama (Tokyo Bay).......... & 20 & 20 & 4 & 20 & 9 & 94 \\
\hline Maézaka (Hamana Lake)........ & 20 & 16 & 0 & 16 & 0 & 38 \\
\hline Yokosuka (Isé Bay) ........... & 20 & 14 & 4 & 14 & 0 & 25 \\
\hline Osaka (Isé Bay)............ & 20 & 20 & 20 & 20 & 324 & 843 \\
\hline Kuwana (Isé Bay) . ............ & 30 & 6 & 1 & 6 & 2 & 2 \\
\hline Hikimoto (Owashi Bay)......... & 20 & 0 & 0 & 0 & 0 & 0 \\
\hline Seno (Kojima Bay)............ & 30 & 0 & 0 & 0 & 0 & 0 \\
\hline Kaidaichi (Kaida Bay).......... & 30 & 0 & 0 & 0 & 0 & 0 \\
\hline Nihoshima (Kaida Bay)......... & 30 & 0 & 0 & 0 & 0 & 0 \\
\hline Kusatsu (Hiroshima Bay)....... & 30 & 0 & 0 & 0 & 0 & 0 \\
\hline Kagami (Yatsushiro Bay)... .... & 10 & 0 & 0 & ${ }^{20} 0$ & 0 & 0 \\
\hline Suminoe (Ariake Bay) ........ & 20 & 0 & 0 & 0 & 0 & 0 \\
\hline
\end{tabular}

Ce parasite se rencontre exclusivement fixé par sa ventouse orale à la face extèrne du manteau. Tout d'abord, la métacercaire s'établit sur la portion antérieure du manteau, généralement du côté gauche, au voisinage de la bouche. Le plus souvent, les individus sont rassemblés par groupes, rapprochés par leur extrémité antérieure, chaque groupe rappelant la disposition d'une fleur de chrysanthème et comprenant de 3 à 90 individus; dans ce dernier cas, le groupe atteint souvent environ $3 \mathrm{~mm}$. de diamètre. On observe aussi des groupes plus grands, mais ils semblent formés de la réunion de plu- 
sieurs petits groupes. Lorsque l'infection est intense, toute la surface du manteau est semée de groupes de parasites. Comme conséquence, la chair de l'huître devient maigre, présentant une coloration grisâtre au lieu de la coloration normale d'un blanc de lait. Même chez une coquille morte, la trace de l'infection est reconnaissable : la face interne est marquée d'aspérités, que provoque toujours la présence du parasite.

Le nombre des parasites semble être influencé par différents facteurs. Tout d'abord la nature du fond où vit l'huitre semble avoir une grande importance. Le tableau précédent montre clairement que sur les fonds de vase de la baie de Tokyo, particulièrement dans la partie ouest (c'est-à-dire à Shinagawa, Omori, Daishigawara, Kanagawa et Yokoha ma), les huîtres sont plus contaminées par ce parasite que dans la partie est (par exemple à Futtsu, Kisarazu, Narawa et Yawata). C'est un fait bien connu qu'un abondant dépôt de vase ne peut éventuellement se former que là où les courants de marée ne sont pas rapides. De tels courants, toutes choses égales d'ailleurs, offrent plus de facilité à la larve du parasite pour s'installer dans son hôte. Comme le parasite s'attache à la face externe du manteau, qui est constamment lavée par l'eau inhalée, la salinité du milieu a certainement une influence directe, parmi les facteurs qui lui permettent de se maintenir vivant. Parmi les localités examinées, Futtsu est la limite méridionale de la rive est de la baie de Tokyo où l'ostréiculture peut être rationnellement pratiquée; en cet endroit, c'est bien rarement seulement que l'eau douce peut avoir quelque influence, en conséquence la densité de l'eau est supérieure à 1,023 à $17^{\circ} 1 / 2 \mathrm{C}$., tandis qu'à Shinagawa où une importante masse d'eau douce est apportée par la rivière Sumida, la densité descend souvent aussi bas que celle de l'eau de la rivière. Pour connaître la durée de la vie du parasite dans des eaux de salinités différentes, j'ai placé dans de tels milieux, pendant cinq heures, un certain nombre de parasites venant d'être détachés de leur hôte. Dans l'eau douce, comme dans celle de faible densité, le parasite ne présente pas de changement alors qu'il périt en un temps comparativement court dans des eaux à densité élevée, ce qu'indique le tableau suivant :

Tableau II. - Relation entre la densité de l'eau et la durée de la vie du distome

\begin{tabular}{|c|c|c|}
\hline Densité de l'eau & Durée de la vie & Température de l'eau \\
\hline 1.024 & 5 heures & $20^{\circ} \mathrm{C}$ \\
\hline $1.026 \ldots \ldots \ldots$ & 30 minutes & - \\
\hline $1.028 \ldots$ & $15-$ & - \\
\hline $1.030 \ldots \ldots \ldots \ldots \ldots$ & $10-$ & - \\
\hline
\end{tabular}


. Cette simple expérience suffit pour permettre de dire que le parasite se porte bien dans les eaux qui sont le mieux appréciées pour la culture de l'huître. D'où il résulte qu'il est bien difficile de l'exterminer dans une telle eau et spécialement ainsi entièrement abrité sous la coquille.

Le nombre des parasites varie naturellement avec l'âge de l'hôte, ou, en d'autres termes, avec le temps depuis lequel il est immergé. Pour vérifier le fait, j'ai examiné un certain nombre d'huîtres de différents âges, élevées die la même manière, sur le même fond. Le résultat a été que les parasites ont été trouvés en nombres extrêmement différents, ainsi que le montre le tableau :

Tableau III. - Nombre moyen de distomes dans vingt huitres examinées à Yawata (Préfecture de Chiba)

\begin{tabular}{|c|c|c|}
\hline Age de l'hôte & Côté droit du manteau & Côté gauche du manteau \\
\hline 1 an.......... & 27 & 84 \\
\hline 2 ans......... & 235 & 1.026 \\
\hline
\end{tabular}

Toutefois, une aussi grande différence n'est pas observable dans tous les cas, bien que l'augmentation du nombre des parasites avec l'âge, soit un fait indéniable. Il y a plusieurs années, j’ai posé une tuile collecteur de naissain d'huître sur le banc, à Yawata. Je l'ai laissée de mai à octobre et constamment examinée. Chez le naissain venant de se fixer sur la tuile, de bonne heure en mai, il n'a jamais été constaté la moindre trace de parasite. Mais, déjà en juin, le naissain ayant acquis une taille d'environ $10 \mathrm{~mm}$. de diamètre, un petit nombre de distomes étaient visibles sur le côté gauche du manteau de l'hôte. Un peu plus tard, en octobre, très peu de naissains s'étaient encore attachés à la tuile, mais ils sont restés constamment exempts de parasites et ainsi jusqu'au printemps suivant. Chaque année, au printemps et particulièrement au début de l'été, une nouvelle infection des huîtres par les cercaires de ce distome était constatée.

Le nombre de distomes en relation avec le temps que l'hôte a passé immergé est aussi expliqué par la comparaison des hauteurs auxquelles les tuiles collectrices sont au-dessus du fond, avec le nombre de parasites dans les huîtres fixées respectivement sur chaque tuile.

Tableau IV. - Nombre moyen de distomes dans dix huitres sur les deux faces des tuiles à différentes hauteurs

\begin{tabular}{|c|c|c|c|}
\hline Hauteur de la tuile collectrice & Face supérieure & Face inférieure & Total \\
\hline $5 \operatorname{sun}(1) \ldots \ldots \ldots \ldots$ & 23 & 236 & 259 \\
\hline 10 sun $\ldots \ldots \ldots \ldots \ldots$ & 7 & 150 & 157 \\
\hline $15 \operatorname{sun} \ldots \ldots \ldots \ldots \ldots$ & 6 & 14 & 20 \\
\hline
\end{tabular}

(1) 1 sun $=3 \mathrm{~cm} ., 03$. 
Ce tableau montre clairement que le parasite a une tendance marquée à se diriger vers l'obscurité. Cela est vraisemblablement dû à son comportement particulier, exactement comme le naissain d'huître fuit les emplacements éclairés et se fixe sur la face obscure de la tuile. On peut donc presque affirmer que les parasites deviennent de plus en plus nombreux à mesure que l'hôte est plus rapproché du fond. A tel point que les huîtres qui sont complètement ensevelies dans les fonds sales et vaseux ou à surface non entretenue, ont le manteau intensément attaqué par le parasite, ce qui provoque une sérieuse inflammation de la chair. A Hiroshima, le traitement habituel du banc d'huîtres, pendant l'été, consiste en un fréquent râclage, car on estime, comme il se doit, que cette manipulation doit amener une croissance uniforme des huitres. Si la même pratique était adoptée dans la baie de Tokio, non seulement elle rendrait l'huître elle-même plus vigoureuse, mais, en même temps, elle préviendrait sûrement la contamination par le parasite. Une expérience destinée à vérifier cette prévision a été conduite, il y a quelques années, à Kisarazu.

Tableau V. - Différence dans le nombre de distomes chez vingt huîtres d'un fond râclé et d'un fond non râclé

\begin{tabular}{|c|c|c|}
\hline Fond & Nombre d'huîtres parasitées & Nombre moyen dè distomes dans une huitre \\
\cline { 1 - 3 } Râclé .................. & 18 & 62 \\
Non râclé..... & 20 & 402 \\
\hline
\end{tabular}

Il est, pour le présent, hors des possibilités de guérir cette maladie parasitaire, mais sa prévention peut être poursuivie sur une certaine échelle. La mesure la plus efficace n'est pas de faire une sélection, pour l'exploitation des bancs d'huitres, des localités, selon leur degré de susceptibilité à être atteintes de cette maladie ; en effet, si les autres conditions sont, en un endroit suspect, favorables en toute assurance à la pose des collecteurs de naissain, on n'aura qu'à placer ces derniers à une certaine hauteur au-dessus du fond. Aussitôt le naissain détaché, il devra être répandu sur un fond baigné par des eaux courantes, qui sera râclé aussi fréquemment que possible.

Note SUR L'ESPÈce PRÉcÉdente ET DIAgnose. - A première vue on pourrait être tenté de prendre cette métacercaire pour une larve de Gymnophallus, non seulement en raison de son aspect général, mais encore de son habitat. Comme les métacercaires de Gymnophallus, elle se trouve parasite " inter pallium et conchas " du mollusque deuxième hôte, généralement par groupes de quelques individus, sans sécrétion de kyste. On retrouve la même disposition 
des ventouses, du pharynx, des cæca intestinaux, même emplacement et même disposition sensiblement symétrique des testicules et des vitellogènes compacts ; position analogue - bien qu'un peu plus antérieure - de l'ovaire, en avant d'un .des testicules ; même vessie en lyre à longues branches. Jusque dans les structures, si nous laissons de côté la question du receptaculum seminis, les analogies sont étroites, ainsi qu'il résulte de la comparaison avec les descriptions de larves ou d'adultes de Gymnophallus, publiées par exemple par Levinsen (1881), H. Lyster Jameson (1902), Odhner (1900 et 1905), M.-V. Lebour (1908 et 1912), R.-Ph. Dollfus (1911, 1912, 1923), H. Lyster Jameson et W. Nicoll (1913), etc...

Un caractère cependant, sépare manifestement la larve de Fujita des vrais Gymnophallus; le pore génital est bien aussi situé sur la ligne médiane, mais, au lieu d'être exactement contigu au bord antérieur de l'ouverture de la ventouse ventrale, il est à une certaine distance en avant de la ventouse.

Nous nous sommes demandé si, chez les Gymnophallus typiques n'ayant pas encore atteint leur état parfait, le pore génital, avant de devenir fonctionnel, n'occupait pas un emplacement plus antérieur que l'emplacement définitif. Quelques métacercaires considérées comme appartenant au genre Gymnophallus; sont en effet connues, chez lesquels il existe, nettement en avant de la ventouse ventrale, l'indication d'un orifice ovale transverse, qui correspond bien à l'orifice génital. La comparaison de Metacercaria Gymnophallus macomæ Marie-V. Lebour (1908, p. 6-7, 20, pl. I, fig. 2 ; 1912, p. 429, 454, pl. XXV, fig. 11), de Macoma balthica L., des côtes du Northumberland, avec la métacercaire de Fujita, est particulièrement importante à ce sujet.

Toutefois, l'examen des coupes de la métacercaire de Fujita, déjà très avancée dans son développement, montre que l'orifice génital (bien que pas encore fonctionnel) correspond à une entaille très profonde, celle-ci est trop distante de la ventouse pour que l'on puisse prévoir, au cours de la croissance ultérieure, une diminution de l'espace les séparant, suffisante pour l'amener au même emplacement que chez les Gymnophallus. Nous croyons donc être en droit de proposer l'adoption provisoire d'un genre nouveau pour la métacercaire de Fujita, en attendant de connaître la forme définitive (vraisemblablement très peu différente), sexuellement mûre et parfaitement adulte, qu'elle acquiert dans l'oiseau de mer, hôte définitif.

Si nous laissons de côté, dans l'état actuel de nos connaissances, la question de l'existence du receptaculum seminis, le nouveau genre : Gymnophalloides nobis, aura comme principale caractéris- 
tique, permettant de le séparer de Gymnophallus, celui de l'emplacement du pore génital, à une certaine distance en avant de la ventouse ventrale, sur la ligne médiane.

Ajoutons que nous avons personnellement étudié une autre métacercaire paraissant susceptible d'être rapportée au genre Gymnophalloides, mais nous n'en avons pas encore publié la description. Metacercaria Gymnophalloides tapetis nobis est parasite " inter pallium et conchas ", par groupe de quelques individudus, chez les Tapes pullaster Montagu, de Saint-Vaast-la-Hougue (Manche). C'est une forme qui ne parvient pas à un degré de développement aussi avancé que la métacercaire de Fujita, bien que dans la même position ; elle mesure, après fixation, environ $0 \mathrm{~mm}$., 2 de long, sur $0 \mathrm{~mm}$., 15 de large, la ventouse orale, protractile, a environ $0 \mathrm{~mm}$., 06 sur $0 \mathrm{~mm}$., 045 et la ventouse ventrale $0 \mathrm{~mm}$., 03 sur $0 \mathrm{~mm}$., 02 ; le pharynx a un diamètre de $0 \mathrm{~mm}$., 017, la cuticule est spinulée. A une certaine distance en avant de la ventouse ventrale, sur la ligne médiane, on observe une papille en relief, au centre de laquelle se trouve l'orifice génital non fonctionnel. Une métacercaire très voisine, parasite de Mytilus edulis L. et Venus sp. ? de la baie de Sébastopol, a été décrite par D.-Th. Sinitzine (1911 p. 38-39, 126 pl. V, fig. 80-81) sous le nom d'Adolescaria perla Sin.

Diagnose de Metacercaria: Gymnophalloides tokiensis n. g., n. sp.

Corps à contours ovalaire, arrondi antérieurement, un peu atténué postérieurement, moyennement épais, légèrement aplati ventralement. Cuticule ornée sur toute sa surface, sauf sur les ventouses, de ponctuations en relief, serrées, disposées en quinconces. Parenchyme renfermant de nombreuses grandes cellules apparemment glandulaires. Ventouse orale subterminale, plus grande et plus puissante que la ventrale, qui est située dans la seconde moitié du corps. Pas de prépharynx. Pharynx globuleux, relativement petit. CEsophage de même longueur. Cæca intestinaux courts et renflés, dépassant un peu le niveau du milieu de la longueur du corps. Vessie en V, à larges branches atteignant la ventouse orale. Pas d'organe copulateur. Sinus génital en entonnoir, s'ouvrant par une fente transversale un peu en arrière du milieu de la longueur du corps, au niveau de l'arrière-fond des cæca intestinaux. Pars prostatica très développée, libre dans le parenchyme, claviforme, s'étendant en direction postéro-dorsale dans le plan médio-sagittal, et s'abouchant à la vésicule séminale, sac ovale plus rapproché de la face dorsale et également libre dans le parenchyme. Testicules glo- 
buleux, latéro-ventraux, à peu près symétriques de part et d'autre de la ventouse ventrale. Ovaire globuleux, à peu près de même taille, situé en avant du testicule gauche, au niveau du cæcum intestinal gauche et latéro-dorsalement. Receptaculum seminis présent dans le plan médio-sagittal, près de la ventouse ventrale, en forme de sac ovale, s'ouvrant à la face ventrale de l'ootype (1) et donnant issue au canal de Laurer qui, par un trajet postéro-dorsal, va s'ouvrir sur la face dorsale du corps. Vitellogènes rapprochés de la face dorsale du corps, formant, symétriquement, de chaque côté du plan médiosagittal, une masse compacte, au niveau de la ventouse ventrale, et de l'ootype. Utérus encore peu développé, ne dépassant pas, antérieurement le niveau du fond des cæca intestinaux. Le parasite, à ce stade, sans sécréter de kyste, vit par groupes d'individus, à la surface du manteau d'Ostrea gigas Thunberg, dans la baie de Tokio et la baie d'Isé (Japon). La cercaire habitant le mollusque premier hôte et l'adulte sexué habitant l'oiseau, hôte définitif, sont inconnus.

\section{B. Forme parasite dans la glande génitale}

Metacercaria : Proctœces ostreæ, n. sp. (fig. 4-7)

Espèce de forme allongée, s'atténuant antérieurement, se terminant brusquement à l'extrémité postérieure. La taille est sensiblement plus grande que pour l'espèce précédente ; les grands individus atteignent jusqu'à $3 \mathrm{~mm}$. de long; c'est d'après un spécimen de cette taille que les mensurations ci-dessous des organes ont été effectuées. La plus grande largeur est $0 \mathrm{~mm}$., 97, au niveau de la ventouse ventrale, qui est située dans le deuxième quart de la longueur totale du corps. La cuticule est entièrement inerme mais montre de fines rides transverses, disposées irrégulièrement; toutefois ces rides ne sont ni assez fortes, ni assez profondes pour pouvoir excéder, sur les coupes, $0 \mathrm{~mm}$., 0012 de haut, dans l'épaisseur du tégument.

La ventouse orale est petite, globuleuse, avec un diamètre de $0 \mathrm{~mm}$., 27. La ventouse ventrale est plus grande, ellipsoïdale, allongée transversalement, elle mesure $0 \mathrm{~mm} ., 47$ sur $0 \mathrm{~mm}$., 42 ; elle est distante de 0 mm., 8 environ de l'extrémité antérieure du corps. La paroi des ventouses est épaisse de $0 \mathrm{~mm}$., 075, garnie d'une épaisse

(1) Rappelons qu'il nous a été impossible de reconnaître, sur les préparations que nous avons examinées, si l’organe considéré comme receptaculum seminis par Fujita a droit à cette appellation; nous en tenons cependant compte dans cette diagnose provisoire. 
cuticule. Le prépharynx est extrêmement court(1), le pharynx mesure 0 mm., 18 sur 0 mm., 15 . Il n'y a pas d'œsophage (2), le pha-

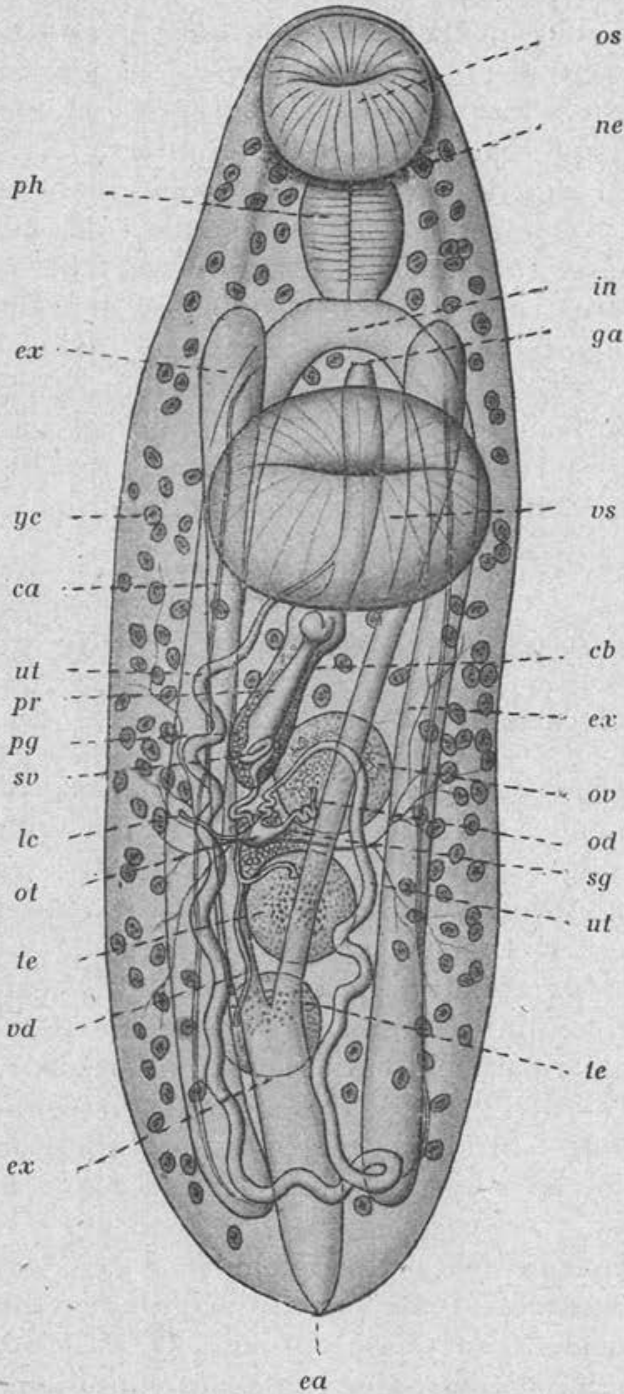

Fig. 4. - Metacercaria: Proctoces ostrea n. sp., individu in loto (demi-diagrammatique), $\times$ env. 80 (mêmes lettres que fig. 1).

(1) Sur un spécimen que nous avons sous les yeux le prépharynx est bien visible et transversalement très large. - R.-Pli. D.

(2) L'examen d'une préparation que nous a communiquée Fujita nous permet de dire que l'œsophage, bien que très court, est nettement présent. - R.-Ph. D. 
rynx est directement en communication avec les branches intestinales, celles-ci commencent à environ $0 \mathrm{~mm}$., 45 de l'extrémité antérieure du corps, elles sont très longues et mesurent $2 \mathrm{~mm}$., 35 ; elles

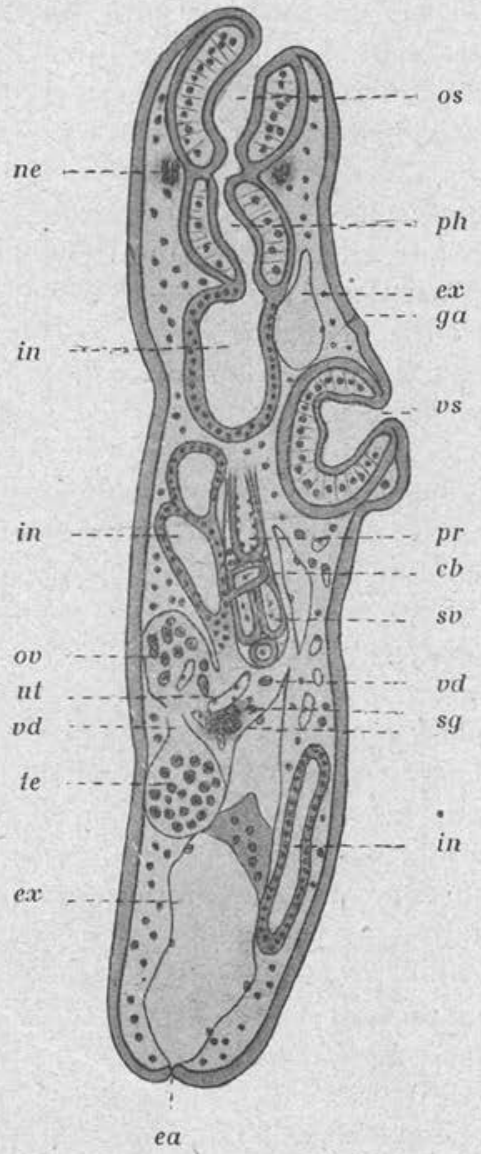

Fig. 5. - Metacercaria : Proctoeces ostrea. Coupe longitudinale, $\times 80$ (mêmes lettres que fig. 1).

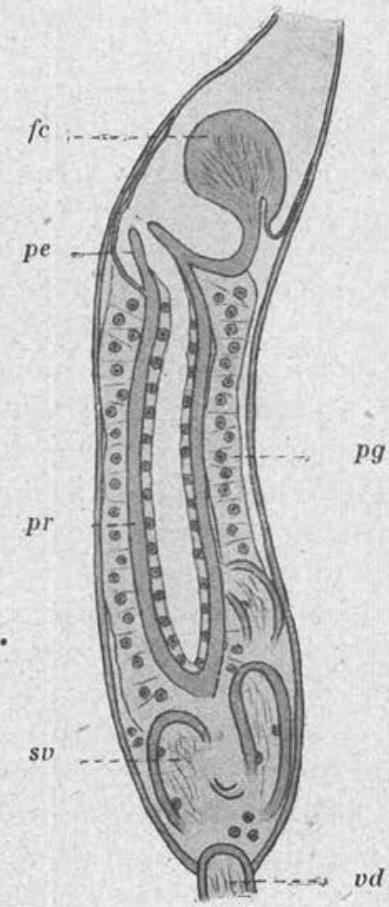

Fig. 6. - Metacercaria : Proctoces ostrex. Poche du cirrhe montrant la crête fibreuse du pénis, $\times 220$ (mêmes lettres que fig. 1).

s'étendent en ligne directe, parallèlement aux deux côtés du corps, distantes entre elles de $0 \mathrm{~mm}$., 44 ; leur extrémité aveugle atteint presque l'extrémité postérieure du corps. En coupe transversale, leur lumière est circulaire et mesure $0 \mathrm{~mm}$., 2. Leur paroi est formée de petites cellules cubiques, hautes d'environ $0 \mathrm{~mm}$., 009. La 
vessie excrétrice est en $\mathrm{Y}$, avec un contour presque parfaitement régulier, ses deux branches se dirigent antérieurement le long du côté ventral des cæca intestinaux et finalement atteignent presque le niveau de l'extrémité postérieure du pharynx ; elles sont élancées, ayant une longueur de $2 \mathrm{~mm}$., 4 sur une largeur de $0 \mathrm{~mm}$., 2 dans leur partie moyenne. Postérieurement, leur réunion forme la partie impaire de la vessie, à $0 \mathrm{~mm}$., 9 de l'extrémité postérieure du corps. Cette partie impaire est relativement courte, rectiligne et large (largeur $0 \mathrm{~mm} ., 3$ ), elle s'ouvre à l'extrémité postérieure du corps. Lès branches donnent irrégulièrement des canaux fins et nombreux. La paroi de la vessie consiste en une très mince membrane pourvue de noyaux et de corpuscules d'excrétion, en particulier dans la paroi des rameaux. En outre, il y a une paire de tubes simples et très fins, de signification énigmatique, courant le long du côté ventral des branches intestinales (fig. $4 c \boldsymbol{c}$ ), à première vue ils semblent être des branches de la vessie, néanmoins ils n'ont aucune communication directe avec elle et n'émettent aucune ramification. De plus, leur structure est totalement différente de celle de cet organe, leur paroi interne étant d'une nature fibreuse d'extrême finesse ; en outre, on n'y voit aucun corpuscule ni rien de similaire susceptible de remplir une fonction d'excrétion (1).

L'appareil génital est compris en grande partie dans le troisième quart de la longueur du corps. Le pore génital, à la face ventrale, s'ouvre sur la ligne médiane (2), à environ $0 \mathrm{~mm}$., 7 de l'extrémité antérieure du corps, juste en avant de la ventouse ventrale ; l'ouverture est très petite, mesuirant $0 \mathrm{~mm}$., 05 dans son plus grand axe. L'atrium génital est un tube conique dont la paroi, modérément épaisse et de nature musculaire, ne renferme qu'un petit nombre de noyaux ; il est long de $0 \mathrm{~mm}$., 25 et large de $0 \mathrm{~mm}$., 04 .

Les deux testicules sont situés l'un devant l'autre, dans le plan médiosagittal et rapprochés de la face dorsale. L’antérieur a son centre à égale distance du centre de la ventouse ventrale et de l'extrémité postérieure du corps ; le postérieur est presque immé-

(1) Ces deux canaux n'ont rien d'énigmatique ; contrairement à l'opinion de Fujita, nous estimons évident que ce sont des canaux excréteurs; ce sont les "gros canaux " ou collecteurs, à trajet récurrent, qui se jettent, un de chaque côté, dans l'arrière-fond de la vessie. Ces collecteurs recoivent les canaux fins venant des ampoules à flamme vibratile et leur paroi n'a évidemment pas la même structure que celle de la vessie.

Etant donnée l'impossibité à laquelle on se heurte, dans la plupart des cas, à suivre dans son ensemble le trajet de ces canaux (et à voir les ramifications), il est admissible que leur aboutissement à la vessie n'ait pu être reconnu par T. Fujita. - R.-Ph. D.

(2) Sur la préparation que nous avons examinée le pore génital est un peu à gauche de la ligne médiane. 
diatement en arrière de lui ; tous deux sont également globuleux, ovalaires, mais pas tout à fait de même taille, l'antérieur étant légèrement plus petit et mesurant $0 \mathrm{~mm}$., 17 sur $0 \mathrm{~mm}$., 13. Les spermiductes partent du côté antéro-ventral des testicules; ils se dirigent antérieurement et se réunissent en un canal commun, un peu en arrière de l'ootype. A partir de cet organe, le canal se dilate légèrement et, après avoir formé une boucle, s'ouvre dans la vésicule séminale, à l'intérieur de la poche du cirrhe. Sa longueur est de $0 \mathrm{~mm}$., 33, sur une très faible largeur : $0 \mathrm{~mm}$., 004 ; sa paroi est très épaisse, avec peu de noyaux. La poche du cirrhe est un tube

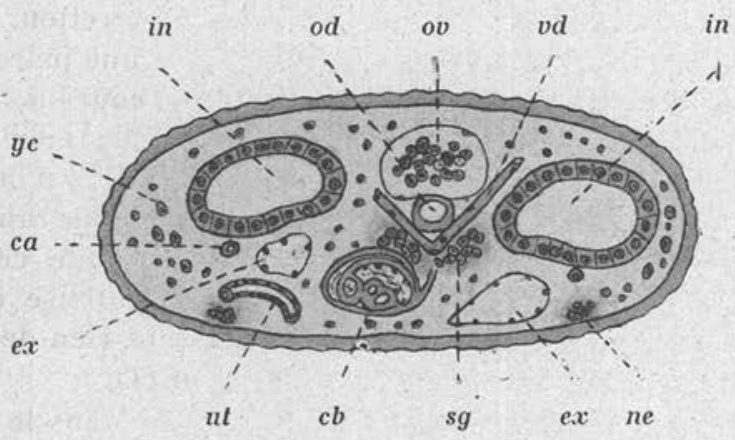

Fig. 7. - Metacercaria : Proctoces ostrex. Coupe transversale montrant une partie de l'appareil génital (mêmes lettres que fig. 1).

conique très allongé, renflé dans sa partie postérieure; elle est longue de $0 \mathrm{~mm}$., 5 environ, sa plus grande largeur est de $0 \mathrm{~mm}$., 06 ; elle est située à moitié chemin entre la ventouse ventrale et le testicule antérieur et elle s'étend obliquement de la région centrale moyenne vers la région dorsale. Sa paroi n'est pas épaisse, mais de nature fibreuse. La vésicule séminale est un tube étroit de $0 \mathrm{~mm}$., 012 de diamètre, contourné en $\mathrm{S}$; sa paroi est mince et fibreuse, haute d'environ $0 \mathrm{~mm}$., 003. Antérieurement, il existe une constriction bien distincte, au delà de laquelle la paroi du tube est en plus garnie d'une membrane avec nombreux noyaux. Cette portion est la prostate qui, en coupe, est presque parfaitement ronde avec un diamètre de $0 \mathrm{~mm}$., 026 ; elle est extérieurement entourée de nombreuses cellules prostatiques. La prostate se continue par le pénis, qui est court et conique, avec une paroi très épaisse, fibreuse et assez pauvre en noyaux; il mesure environ $0 \mathrm{~mm}$., 05 sur $0 \mathrm{~mm}$., 03 . Son extrémité est pourvue d'une crête à structure fibreuse homogène. Cette crête s'attache au cirrhe par deux suspenseurs dont la 
contraction sert sans doute à la protrusion du cirrhe au dehors.

L'ovaire est parfaitement sphérique et mesure $0 \mathrm{~mm}$., 11 sur $0 \mathrm{~mm}$., 07. Il est situé à environ $0 \mathrm{~mm} ., 4 \mathrm{du}$ bord postérieur de la ventouse ventrale et exactement antéro-dorsal par rapport au testicule antérieur. L'oviducte part de son côté dorsal et, après un petit nombre de circonvolutions, se dirige postérieurement en arrière, vers l'ootype, dans la partie médio-centrale du corps. Cet oviducte est court et à paroi mince, mais c'est un tube relativement large, ayant un diamètre de $0 \mathrm{~mm}$., 013. L'ootype a une forme sphérique, avec une membrane interne nucléée; son diamètre est d'environ $0 \mathrm{~mm}$., 03. L'utérus part de son côté dorsal et, après quelques sinuosités, se dirige antérieurement jusqu'au niveau de l'ovaire ; à ce niveau, s'élargissant plus ou moins, il descend postérieurement jusqu'à l'extrémité distale de l'intestin, où, par un trajet horizontal transverse, il atteint l'autre côté du corps et remonte antérieurement le long de la branche droite de la vessie. A proximité de la ventouse ventrale, il diminue graduellement de largeur et devient le vagin. La longueur de l'utérus atteint ainsi près de $5 \mathrm{~mm}$., avec une lumière de $0 \mathrm{~mm}$., 008 de diamètre. Sa paroi est épaisse et multinucléée.

L'œuf a une forme ovale, il mesure $0 \mathrm{~mm}$., 04 sur $0 \mathrm{~mm}$., 02, la coque est mince. La glande coquillière est représentée par un massif irrégulier de cellules rassemblées autour de l'ootype. Il n'y a pas de receptaculum seminis. Le canal de Laurer s'ouvre du côté dorsal dans l'ootype d'une part et à la face dorsale du corps d'autre part. Les vitellogènes sont constitués far des follicules glandulaires disposés sans ondre dans les parties latéro-dorsales du corps ; ils s'étendent antérieurement jusqu'à la région pharyngienne et postérieurement jusqu'à l'extrémité de l'intestin(1). Les vitelloductes

(1) A l'inverse de Fujita nous n'admettons pas que les nombreuses grandes cellules apparemment glandulaires dispersées dans le parenchyme d'une extrémité à l'autre du corps, soient des follicules vitellogènes. Il est possible qu'à ce stade les vitellogènes ne soient pas encore bien nettement distincts. Sur un spécimen coloré que nous avons examiné, nous n'avons pu les reconnaître avec certitude. Il est vraisemblable toutefois que les follicules vitellogènes, restent confinés latéralement dans le troisième quart de la longueur du corps

Rappelons que Looss (1901, p. 404), dans sa description du Proctaces maculatus (Looss) des labridés, a attiré l'attention sur les nombreuses grandes cellules apparemment glandulaires remplissant le parenchyme; il a insisté sur le fait qu'élles simulent des vitellogènes, en particulier dans les parties latérales de la région antérieure du corps et qu'elles peuvent être facilement confondues avec eux, faisant croire que ces organes sont beaucoup plus étendus qu'ils ne le sont en réalité. Un examen attentif à un fort grossissement lui a montré que les éléments composant les follicules des glandes vitellogènes n'étaient répartis que sur une faible hauteur, dépassant peu, ou pas, en avant, le niyeau du bord antérieur de l'ovaire, en arrière le niveau du bord postérieur du testicule postérieur. Ces cellules glandulaires du parenchyme ont aussi été étudiées par Odhner (1911, p. 109) qui les a désignées sous le nom de " subcuticularen Drüsenzellen ». R.-Ph. D. 
venant de chacun des côtés du corps finissent par prendre une direction horizontale au niveau de l'ootype, dans les côtés latéraux duquel ils viennent s'ouvrir.

Remarque: L'habitat du présent distome est actuellement cantonné étroitement dans la préfecture d'Hiroshima. En 1904, j'ai récolté et examiné avec soin plusieurs centaines d'huîtres de trois lieux d'exploitations ostréicoles, bien connus dans cette préfecture : ceux de Kaidaichi, Nihoshima et Kusatu. J'ai bientôt reconnu qu'un certain nombre d'entre elles hébergeaient ce parasite dans leur glande génitale. Le degré de leur infection était naturellement variable selon les conditions particulières des localités ci-dessus mentionnées.

Les fonds de Kusatsu sont, dans leur généralité, d'argile sableuse, tandis que ceux de Nihoshima et Kaidaichi sont purement vaseux. Comme conséquence, les huîtres de Kusatsu étaient moins parasitées (proportion de $100 / 0$ ) que celles de Nihoshima (proportion double); celles de Kaidaichi l'étáient à un degré exactement intermédiaire. L'huître infectée ne présente, au début, aucun symptôme apparent permettant de la distinguer de celles dont la condition est normale. Une attention vigilante, cependant, révèle bientôt la présence du parasite. Se détachant sur la couleur générale blanchâtre de la glande génitale, on observe de petites taches, tantôt oblongues, tantôt rondes, généralement d'un blanc crémeux, avec une bordure légèrement grisâtre. Souvent ces taches sont teintées de jaunâtre, ce qui a attiré plus facilement notre attention. Le nombre de ces taches varie d'une à dix, mais plus généralement, il est de deux par hôte. Le nombre maximum de dix a été noté chez 10 0/0 environ de la totalité des huîtres malades examinées. Chaque tache correspond toujours à un distome profondément enfoncé dans le tissu de la glande génitale; il se maintient en position en se fixant à la paroi de l'organe par sa ventouse ventrale. Comme conséquence, la présence de ce distome a souvent un effet désastreux sur l'hôte : elle peut amener l'annihilation totale de sa capacité de reproduction.

Note SUR L'ESPÈCE PRÉCÉdente ET diAgNose. — Cette forme, par l'ensemble de ses caractères, appartient typiquement au genre Proctoces Odhner (1911, p. 107-108), de Ia sous-famille des Haplocladinæ Odhner (1911, p. 103) et de la famille des Steringophoridæ Odhner (1911, p. 98-99).

Bien que, chez l'huître, cette forme présente un début précoce d'activité génitale (1), elle ne paraît pas avoir atteint sa maturité

(1) Dans un exemplaire nous avons compté environ douze œufs ; il ne s'agissait apparemment pas d'œufs normaux, ils étaient de tailles et de formes très diverses, les plus grands mesuraient seulement environ $19 \mu 3 \times 14,19 \mu 3 \times 12,6,17 \mu 3 \times 13,3$. Ils êtaient donc bien plus petits que ceux mesurés par Fujita chez d'autres individús 
complète ni sa taille définitive ; il s'agit d'une métacercaire progénétique se trouvant dans le deuxième hôte intermédiaire du cycle de l'espèce. On ne connaissait pas, jusqu'à présent, le stade métacercaria des Proctoeces ; nous savons maintenant que ce stade se passe sans enkystement. La cercaire correspondante, qui naît dans le Mollusque premier hôte, est inconnue, de même que l'adulte habitant le poisson hôte définitif. Il n'y a, actuellement, qu'une seule espèce de cercaire qui ait été rapportée au genre Proctoeces. Odhne: (1911, p. 108) a supposé que Cercaria setifera J. Müller in La Valette Saint-Georges (1855, p. 38, Pl. II, fig. II) [nec Claparède 1863, nec Villot 1879, nec Monticelli 1888, nec Pelseneer 1906] correspondait à Proctoeces maculatus (Looss sp., 1901, p. 402-404, fig. 3), type du genre Proctoeces Odhner (1911, p. 106, p. 107-108, diagnose).

$P$. maculatus Looss a été trouvé par Looss à Trieste dans la dernière moitié de l'intestin et presque exclusivement dans le rectum, chez Labrus merula L., Crenilabrus pavo C. V., Crenilabrus griseus L. ; il a été retrouvé par Odhner (1911) à Naples chez Blennius ocellaris $\mathrm{L}$.

Une seconde espèce du genre est $P$. erythraeus Odhner (1911, p. 108-110) trouvé à maturité chez Chrysophrys bifasciata Forskal et immature chez Julis lunaris Linné à Tor, mer Rouge, par Odhner.

La métacercaire étudiée par Fujita correspond-elle à une de ces deux espèces ? Il est possible d'écarter tout de suite la seconde, qui a une ventouse ventrale relativement petite (au moins d'un tiers plus petite que chez $P$. maculatus (Looss), dit Odhner), mais la comparaison avec la première montre une ressemblance des plus étroites. Si nous laissons de côté le caractère de l'extension des vitellogènes (caractère que nous ne connaissons pas exactement pour la forme de Fujita) et le caractère de la taille des œufs (ils mesurent $0 \mathrm{~mm}$., 072 à $0 \mathrm{~mm}$., 079 , sur env. $0,027 \mathrm{chez}$ Proct. maculatus (Looss) et seulement 0,04 sur 0,02 chez la métacercaire de Fujita), tous les autres caractères sont concordants, à tel point que les diagnose et description de l'espèce maculatus, par Looss et Odhner, pourraient convenir presque exactement à l'espèce ostreæ Fujita.

Faute de matériaux de comparaison suffisants, nous ne nous croyons pas en droit de conclure à l'idendité des deux formes, mais

et il est très possible que les œufs mesurés par Fujita n'aient pas non plus été des œufs normaux. L'on sait, en effet, que, chez la même espèce, les œufs produits par les individus ayant atteint leur complet développement dans l'hôte définitif peuvent différer tant par leurs plus grandes dimensions que par leur contenu, des oufs précoses produits par les métacercaires progénétiques au début de l'activité génitale. R.-Ph. D. 
nous ne la tenons pas pour inadmissible. Il est à souhaiter que des recherches chez les poissons mangeurs de Lamellibranches, sur les côtes de la préfecture d'Hiroshima, permettent de découvrir des exemplaires complètement adultes de Proctœes ostreæ Fuj., chez lesquels l'extension des vitellogènes et les dimensions des œufs puissent être observées avec précision; il sera alors possible de savoir définitivement si $P$. ostreæ Fuj. doit ou non tomber en synonymie avec $P$. maculatus (Looss). Ajoutons que, selon nous, le seul fait qu'une espèce habite le Japon ne peut pas être invoqué pour dire qu'elle doit être différente, toutes choses égales d'ailleurs, d'une espèce d'Europe. Parmi les Trématodes de la faune du Japon, il y en a un certain nombre qui existent aussi dans la faune de l'Europe occidentale; je rappellerai que Cercaria pectinata Huet (famille des Steringophoridæ), parasite de Tapes (et d'autres Lamellibranches), sur la côte atlantique de France est aussi parasite de Tapes au Japon, où l'a retrouvé Fujita $(1906,1907)$.

\section{Diagnose de Metacercaria : Proctœces ostreæ n. sp.}

Corps allongé, diminuant peu de largeur dans sa partie postérieure, un peu aplati, à cuticule inerme. Parenchyme renfermant de nombreuses glandes unicellulaires dispersées. Ventouses puissantes, assez rapprochées l'une de l'autre, ventouse orale globuleuse, ventouse ventrale beaucoup plus grande, allongée transversalement, proéminente. Prépharynx extrêmement court, large. Pharynx grand et puissant. Esophage extrêmement court. Branches intestinales atteignant presque l'extrémité postérieure du corps. Vessie en Y, la bifurcation est située très peu en arrière du niveau de l'espace intertesticulaire et les branches atteignent le niveau du bord postérieur du pharynx. Pore génital légèrement à gauche de la ligne médiane, non loin du bord antérieur de la ventouse ventrale. Sinus génital très ample, plus ou moins tubulaire. Poche du cirrhe étirée longitudinalement, dorsale par rapport à la ventouse ventrale, plus ou moins parallèle à l'axe du corps, descendant jusqu'à environ le niveau du centre de l'ovaire. Antérieurement elle renferme une pars prostatica cylindriforme assez longue - dont l'épaisse paroi est constituée principalement par une musculature de fibres longitudinales, - et postérieurement une mince vésicule séminale, en forme de boyau, plusieurs fois contournée. L'ouverture de la pars prostatica dans le sinus génital est en forme de museau de tanche et fait fortement saillie, elle est pourvue de chaque côté, d'une crête ou bourrelet de nature fibreuse (ou musculaire?). Testicules glo- 
buleux, à bords entiers, presque tout à fait égaux, situés l'un derrière l'autre, à peine un peu obliquiement (le postérieur est légèrement plus à droite que l'antérieur), et compris à peu près entièrement dans l'avant-dernier cinquième du corps.

En avant du testicule antérieur se trouve l'ovaire, de même forme et presque médian, séparé par une distance peu éloignée de. son diamètre du bord postérieur de la ventouse ventrale. Il n'y a pas de receptaculum seminis, mais il y a un canal de Laurer assez Iono s'ouvrant à la face dorsale. Vitellogènes formés de petits follicules clairsemés (et ne paraissant pas encore avoir atteint leur développement définitif à ce stade), vitelloductes aboutissant horizontalement entre l'ovaire et le testicule antérieur. Utérus développant ses circonvolutions dans la moitié postérieure du corps, s'ouvrant un peu en avant de la pars prostatica dans le sinus génital, par son extrémité distale, insensiblement rétrécie, formant le vagin, mais sans que sa paroi externe acquierre de revêtement cuticulaire. CEufs ovales à coquille mince de couleur jaune, mesurant environ $0 \mathrm{~mm}$., 04 sur $0 \mathrm{~mm}$., 02 , peu nombreux à ce stade metacercaria, où se trouve le parasite, non enkysté, dans la glande génitale d'Ostrea gigas Thunberg, préfecture d'Hiroshima (Japon). Le stade cercaria, parasite dans le mollusque premier hôte, est inconnu, de même que l'adulte parfait parasite dans l'intestin du poisson, hôte définitif.

\section{BIBLIOGRAPHIE}

Dollfus (Robert-Ph.). - L'appareil néphridien de deux cercaires parasites de Donax vittatus Da Costa (C. R. Soc. Biologie. Paris, t. LXXI, 11 nov. 1911, p. $422-425$, fig. 1-4).

- Une métacercaire margaritigène parasite de Donax vittatus Da Costa (Mém. soc. zool. de France, t. XXV, 1912, p. 85-144, fig. texte 1-3. Pl. II, fig. 1-14).

- Le Trématode des perles de nacre des moules de Provence (C. R. Acad. Sc. Paris, t. CLXXVI, 14 mai 1923, p. 1427-1429, 1 fig.).

- Liste critique des cercaires marines à queue sétigère signalées jusqu'à présent (Travaux de la Station zoologique de Wimereux, tome IX, 1925) [en cours d'impression].

- Etudes critiques et documentaires sur quelques trématodes Digenea, avec la description de formes larvaires nouvelles ou peu connues [ouvrage non publié].

Funtra (T.). - Deux espèces de cercaires parasites de l'asari (") (Dobutsugaku Zasshi. Tokyo, t. XVIII, no 214, août 1906, p. 197-203. Pl. V, fig. 1-18) [en japonais].

(") Asari = Tapes philippinarum Adams et Reeve. 
Fujita (T.).- Sur le nom spécifique d'une cercaire parasite de l'asari (Dobutsugaku Zasshi. Tokyo, t. XIV, no 227, sept. 1907, p. 281) [en japonais].

Jameson (Lyster-H.). - On the origin of pearls (Proc. Zool. Soc. London, 1902, vol. I, nos X-XI, March 4, p. 140-166, fig., texte 22-24. Pl. XIV-XVII, fig. 1-16).

JAMESON (Lyster-H.) et NicolL (W.). - On some parasites of the Scoter Duck (Edemia nigra), and their relation to the pearl-inducing Trematode in the edible mussel (Mytilus edulis) (Proc. Zool. Soc. London, 1913, vol. I, March, p. 53-63, fig. texte 11-12.

La Valette Saint-Georges (Ad.-J.-H. DE). - Symbolæ ad Trematodum evolutionis historiam (Dissertatio inaug.). Berolini fo, 38 pages, 2 planches.

Lebour (Marie-V). - Trematodes of the Northumberland coast, ${ }^{\circ}$ II (Trans. Nat. Hist. Soc. of Northumberland, n. s., vol. III, part. I, p. 1-20. PI. I-III).

A review of the British marine cercariæ (Parasitology, vol. IV, $\mathrm{n}^{\circ} 4$, january 8, 1912, p. 416-456. Pl. XXV-XXIX).

Levinsen (G.-M.-R.). - Bidrag til kundskab om Grønlands Trematodfauna (Oversigt over d. k. D. Vidensk. Selsk. Forhdl, 1881, n 1, p. 52-84. Pl. I-III).

Looss (A.). - Ueber einige Distomen der Labriden der Triester Hafens (Centralbl. $f$. Bakt. 1 Abt., XXIX Bd., nos 9-10, 21-28 März 1901, p. 398-405, fig. 1-4, p. 437-442, fig. 1-2).

Odhner (T.). - Gymnophallus, eine neue Gattung von Vogeldistomen (Centralbl. $f$. Bakt. 1 Abt., XXVIII Bd., n² 1, 1900, p. 12-23, fig. 1-4).

- Die Trematoden des arktischen Gebietes (Fauna arclica, IV Bd., 1905, p. 289-372, fig. texte 1-4. Pl. II-IV).

- Zum natürlichen System der digenen Trematoden, III (Zoolog. Anzeiger.. XXXVIII, n॰ 4, 8 Aug. 1911, p. 97-117, fig. 1-8).

Pelseneer (P.). - Trématodes parasites de mollusques marins (Bull. scient. Francè et Belgique, t. XL, 5 e s., p. 161-186. Pl. VIII-XII, fig. 1-54).

Sinitzine (D.-Th.). - La génération parthénogénétique des trématodes et leur progéniture dans les mollusques de la mer Noire (Mém. Acad. imp. sc. St-Pélersbourg, 8e série, Cl. physico-math., vol. XXX, n॰ 5, 1911, 127 pages, fig. texte 1. PI. I-VI).

Hakkaido Imperial University, Sapporo, Japon. Muséum national d'Histoire naturelle, Paris. 